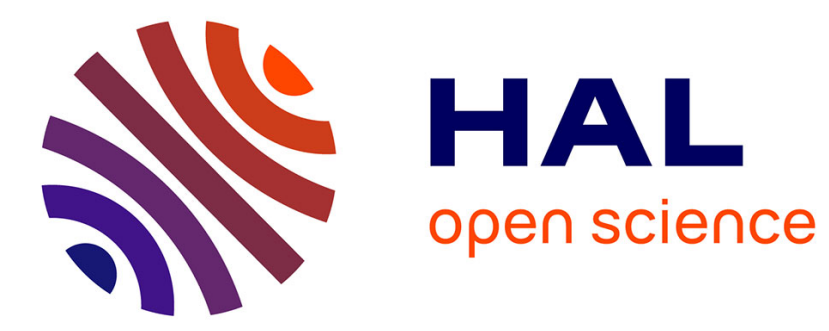

\title{
L'impact de la gestion de la mobilité par les entreprises wallonnes sur les déplacements domicile-travail
}

\author{
Jean-marie Halleux, Marie-caroline Vandermeer
}

\section{To cite this version:}

Jean-marie Halleux, Marie-caroline Vandermeer. L'impact de la gestion de la mobilité par les entreprises wallonnes sur les déplacements domicile-travail. RTS - Recherche Transports Sécurité, 2014, 2014 (02-03), pp.83-100. 10.4074/S0761898014002027 . hal-01670611

\section{HAL Id: hal-01670611 \\ https://hal.science/hal-01670611}

Submitted on 21 Dec 2017

HAL is a multi-disciplinary open access archive for the deposit and dissemination of scientific research documents, whether they are published or not. The documents may come from teaching and research institutions in France or abroad, or from public or private research centers.
L'archive ouverte pluridisciplinaire HAL, est destinée au dépôt et à la diffusion de documents scientifiques de niveau recherche, publiés ou non, émanant des établissements d'enseignement et de recherche français ou étrangers, des laboratoires publics ou privés. 


\title{
L'impact de la gestion de la mobilité par les entreprises wallonnes sur les déplacements domicile-travail
}

\section{The impact of mobility management by Walloon companies on commuting}

\author{
Jean-Marie Halleux · Marie-Caroline Vandermeer
}

Reçu le 29 mars 2013, accepté le 15 janvier 2014

(C) IFSTTAR et Éditions NecPlus 2014

\begin{abstract}
Résumé Bien que la gestion de la mobilité par les entreprises soit une pratique de plus en plus courante, la littérature sur ce sujet ne permet pas de dégager de conclusions bien tranchées quant à l'impact réel des mesures adoptées. Dans cette perspective, cet article cherche à préciser l'impact sur l'utilisation de l'automobile des mesures adoptées par les entreprises situées en Wallonie. Afin d'aboutir à cet objectif, l'article développe des simulations qui précisent comment l'efficacité des mesures interagit avec d'autres déterminants des pratiques modales. En intégrant les mesures en faveur du covoiturage et les mesures en faveur des TC, nous aboutissons à une réduction comprise entre $7,2 \%$ et $8,4 \%$ de la conduite automobile. Pour les mesures en faveur des TC, contrairement à l'hypothèse initiale, l'efficacité apparaît moindre en centre urbain qu'en périphérie. Concernant les mesures en faveur $\mathrm{du}$ vélo, les résultats témoignent d'un report modal qui s'effectue essentiellement depuis la marche plutôt que depuis la voiture.
\end{abstract}

Mots clés Wallonie - report modal - gestion de la mobilité . profil d'accessibilité $\cdot$ covoiturage $\cdot$ déplacement

domicile-travail

\begin{abstract}
Although mobility management by employers is an increasingly common practice, literature is scarce on the real impact of the applied measures. In this perspective, the objective of this paper is to assess the impact on car use of the measures adopted by employers located in Wallonia. In order to achieve this objective, the article develops
\end{abstract}

Jean-Marie Halleux $(\bowtie)$

Département de géographie (B11) - Allée du 6 août, nº 2 - B - 4000

Liège, Belgique

e-mail : jean-marie.halleux@ulg.ac.be

Marie-Caroline Vandermeer $(\bowtie)$

Département de géographie (B11) - Allée du 6 août, n 2 - B - 4000

Liège, Belgique

e-mail : mc.vandermeer@ulg.ac.be simulations that put forward the impact of the measures based on their interrelations with other modal choice factors. Our assessment leads to the conclusion that measures promoting public transport and car-pooling can reduce solo car driving between $7,2 \%$ and $8,4 \%$. In contrast to our assumption, measures promoting public transport are less effective in central areas than in urban outskirts. Regarding measures promoting the use of bicycle, the findings suggest that the modal shift occurs from other modes rather than from car use.

Keywords Wallonia - Modal shift - Mobility management . Accessibility profile $\cdot$ Carpooling $\cdot$ Commuting

\section{Introduction}

Il est aujourd'hui reconnu que des politiques de transport exclusivement fondées sur le développement de l'offre et des infrastructures ne peuvent apporter des réponses durables et efficaces aux défis de la mobilité [1]. En complément de l'intervention sur l'offre, des mesures visant à limiter la demande sont indispensables. L'aménagement du territoire représente ici un levier fondamental, afin de localiser un maximum de logements, d'emplois et d'équipements sur des lieux dont le profil d'accessibilité est favorable à la marche, $\mathrm{au}$ vélo et aux transports en commun, c'est-à-dire aux modes de transport considérés comme des « alternatives » à l'automobile [2].

En plus des solutions techniques et urbanistiques, les politiques de déplacement doivent intégrer des mesures visant à influencer les comportements. La gestion de la mobilité par les entreprises s'inscrit dans ce cadre. Cette gestion vise, pour les firmes privées, mais également pour les institutions publiques (administrations, écoles...), à organiser des actions susceptibles de réduire la conduite automobile de leurs salariés. La mise en place de ces actions passe par la préparation de plans de mobilité d'entreprise, qui permettent d'offrir aux travailleurs un éventail le plus varié possible de mesures susceptibles 
de favoriser le télétravail, le covoiturage et les modes alternatifs à la voiture.

La gestion de la mobilité par les entreprises est une pratique relativement récente. Les premières mesures réglementaires visant à les y encourager furent prises à la fin des années quatre-vingt dans la région de Los Angeles [3]. Cette zone urbaine, archétype d'un mode de vie dépendant de la route et de l'automobile, supporte alors de plus en plus difficilement la paralysie du trafic et les effets négatifs de la pollution atmosphérique due au transport. Diverses mesures y furent introduites, dont le Commuter Program, qui impose aux entreprises de plus de 100 travailleurs de préparer un plan visant à réduire l'autosolisme de leurs salariés, c'est-à-dire le fait de voyager seul en voiture [4]. À partir des années 1990, des initiatives similaires sollicitant l'implication des entreprises voient le jour dans plusieurs pays européens, dont les Pays-Bas, le Royaume-Uni et l'Allemagne [3]. La Commission européenne se préoccupe également du sujet des plans de mobilité d'entreprise, comme l'illustre tant la mise en exergue du rôle des entreprises dans son plan d'action sur la mobilité urbaine [5] que le financement de la plateforme EPOMM dédiée au management de la mobilité [6].

L'attention croissante des entreprises pour la gestion de la mobilité s'est accompagnée d'une attention croissante des chercheurs pour cette problématique. Pour autant, comme nous le détaillons ci-dessous, les méthodes d'évaluation ne sont pas encore très approfondies et les recherches actuelles ne permettent pas de dégager de conclusions bien tranchées quant à l'impact réel des mesures adoptées [6, 7]. L'analyse développée dans cet article cherche à combler cette lacune. Son principal objectif est de quantifier l'impact de la gestion de la mobilité par les entreprises wallonnes sur les parts modales des déplacements domicile-travail. Pour ce faire, nous nous sommes intéressés tant au report vers les modes alternatifs qu'au covoiturage. Vu les données mobilisées, le sujet du télétravail n'a pas pu être considéré et il sort donc du cadre de cet article.

Pour les navettes de travail dont la destination se situe en Wallonie, la part modale de l'automobile (conducteur et passager) est en moyenne supérieure à $80 \%$ [8]. La voiture représente donc, et de loin, un mode de transport très prédominant. Pour autant, comme nous le détaillons ci-dessous, une localisation sur un site suffisamment accessible par d'autres modes ainsi que des mesures actives de gestion de la mobilité permettent de limiter cette utilisation massive de l'automobile.

Notre démarche a consisté dans le développement de simulations qui différencient l'impact des mesures adoptées par les entreprises en fonction de leurs caractéristiques. Parmi ces caractéristiques, nous avons notamment pris en compte leur localisation à une échelle macro et à une échelle micro. Cela a permis de préciser l'impact de la gestion de la mobilité par les entreprises en fonction de l'organisation spatiale des territoires et en fonction de l'accessibilité des lieux par les modes alternatifs. Afin de répondre à la question de l'impact de la gestion de la mobilité par les entreprises, nous avons donc cherché à comprendre comment cet impact interagit avec d'autres déterminants des pratiques modales et, en particulier, avec les déterminants liés aux caractéristiques des environnements spatiaux. Dans le paysage de la recherche sur les préférences modales, nous nous inscrivons dans une tradition qui, complémentairement aux approches micro-économiques et socio-psychologiques, souligne l'importance des contextes spatiaux [9].

La suite de l'article est structurée en quatre parties. La première est une revue de la littérature dédiée à l'impact de la gestion de la mobilité par les entreprises sur les choix modaux de leurs salariés. En deuxième et en troisième parties, nous détaillons les données mobilisées pour nos traitements et la méthodologie adoptée. Enfin, en quatrième partie, nous présentons nos résultats en considérant, d'une part, l'impact des mesures visant à favoriser l'usage des transports collectifs et, d'autre part, l'impact des mesures visant à favoriser les pratiques du covoiturage et du vélo.

\section{Un intérêt croissant des chercheurs. . . mais des méthodes d'évaluation qui demeurent peu approfondies}

Nous venons de souligner que l'attention croissante des entreprises pour la gestion de la mobilité s'est accompagnée d'un intérêt croissant de la part des chercheurs. La littérature disponible sur cette problématique est ainsi de plus en plus conséquente et une synthèse en a récemment été publiée par une équipe rassemblée autour de Vanoutrive [10]. Nous reprenons ci-dessous certains points principaux de cette synthèse. Ensuite, nous focalisons notre propos sur l'évaluation de l'impact des mesures, une problématique relativement peu détaillée par Vanoutrive et ses collègues.

L'état de l'art de Vanoutrive et al. permet notamment de comprendre comment certaines caractéristiques des contextes institutionnels, réglementaires et politiques poussent les entreprises à s'intéresser aux enjeux sociétaux de la mobilité. En particulier, cet état de l'art s'intéresse aux raisons qui, derrière le paravent de l'enjeu collectif de la limitation des trafics automobiles, poussent les entreprises à adopter des mesures visant à modifier les comportements de mobilité de leurs travailleurs [11]. Par exemple, pour le contexte belge, les premiers objectifs des entreprises qui activent le levier de la gestion de la mobilité sont d'accroître le bien-être de leur personnel (et donc leur productivité), ainsi que leur image (tant auprès du monde extérieur qu'auprès de leurs salariés) [12]. 
Les mesures mises en place par les entreprises dans le cadre de leurs plans de mobilité peuvent être répertoriées en deux grandes catégories : celles relatives à l'utilisation de l'automobile d'une part et celles relatives à l'utilisation des alternatives à ce mode prédominant d'autre part. Il faut ensuite discerner les mesures incitatives des mesures dissuasives. Pour les premières, soulignons l'importance des mesures consistant à octroyer un avantage financier aux salariés qui utilisent un mode de transport autre que la conduite individuelle. Dans le cadre des plans de mobilité, ces mesures sont relativement simples à concevoir. Néanmoins, elles peuvent représenter un coût non négligeable pour l'employeur [13]. Les mesures dissuasives se concentrent généralement sur le stationnement, soit en limitant le nombre d'emplacements disponibles, soit en les rendant payants. Les mesures qui agissent sur le stationnement sont souvent efficaces pour limiter la part modale de la voiture. Pour autant, comme Rye et Ison l'ont bien montré, elles sont impopulaires et leur mise en place est dépendante de conditions assez restrictives [14].

Notre revue de la littérature montre que les connaissances actuelles sur l'impact de la gestion de la mobilité par les entreprises découlent de deux types d'approches. Nous trouvons en premier lieu une vaste gamme de recherches sur les déterminants des pratiques modales. Ces recherches peuvent, parmi d'autres aspects, prendre en considération des déterminants sur lesquels les entreprises ont une certaine prise. Toutefois, dans ces travaux, la relation avec la gestion de la mobilité par les entreprises n'est généralement qu'indirecte. En deuxième lieu, nous trouvons des recherches spécifiquement dédiées aux plans de mobilité d'entreprise. Parmi ces recherches, il en existe quelques-unes qui, à l'instar de cet article, se focalisent sur l'évaluation quantifiée des impacts.

À propos des analyses et études sur la problématique générale des choix modaux, faisons référence à une synthèse récente publiée par De Witte et al. [9]. Cette synthèse très fouillée propose une typologie qui série utilement les déterminants. Les quatre groupes suivants y sont différenciés :

- les caractéristiques des déplacements (motifs, distance, durée, chaînage, coût. ..);

- les caractéristiques des environnements spatiaux des déplacements (densité, diversité fonctionnelle, offre en transports collectifs, disponibilité en emplacements de parking. ..);

- les caractéristiques socio-démographiques des individus (âge, niveau d'éducation et de revenu, composition des ménages. ..) ;

- les caractéristiques socio-psychologiques des individus (mode de vie, perception, habitude...).
Présenter cette typologie conduit à rappeler que le rôle des entreprises sur les comportements de mobilité ne se limite pas aux plans visant la promotion d'une mobilité durable. En la matière, nous ne pouvons passer sous silence les deux problématiques majeures du rôle des voitures de société sur le coût des déplacements et du rôle des choix de localisation sur les caractéristiques des environnements spatiaux.

Le sujet des choix de localisation renvoie au domaine de l'aménagement du territoire et aux recherches sur les relations entre transport et formes urbaines (voir par exemple Ewing et Cervero pour une synthèse générale [15] ; Dujardin et al. pour le contexte wallon [16] et Le Néchet et Aguiléra pour le contexte français [17]). Ces recherches dédiées à l'influence des formes urbaines sont à relier à un élément fondamental que ne manque pas de souligner la synthèse de De Witte et al. [9], à savoir l'interdépendance entre déterminants. S'agissant par exemple de l'effet de la densité sur la limitation de la conduite, il est aujourd'hui reconnu qu'il est conditionné par de nombreux facteurs tels que la gestion plus ou moins restrictive du stationnement, l'organisation et la fréquence des transports collectifs ou la distribution spatiale des emplois à l'échelle des régions fonctionnelles [17]. L'interdépendance entre déterminants est également fondamentale pour la problématique du vélo [18], comme en témoigne la synthèse de Pucher et al. sur les politiques en faveur des circulations douces [19]. Dans cette synthèse, la principale conclusion consiste à souligner qu'un développement significatif de la pratique du vélo ne peut apparaître qu'en présence de politiques intégrées où les mesures en faveur des deux-roues prennent appui sur les choix portés en matière d'occupation des sols, d'infrastructures et de restrictions vis-à-vis de l'automobile.

À propos des voitures de société, il est établi que leur effet sur le coût des déplacements pousse à une utilisation abusive de l'automobile (voir par exemple De Witte et al. pour la Belgique [20] ou Kingham et al. pour le Royaume-Uni [21]). L'unanimité de la littérature sur ce sujet témoigne de l'importance des mesures qui impactent les coûts de déplacement des travailleurs [22]. Ces mesures financières sont aussi mises en exergue pour les initiatives qui avantagent les salariés utilisant un mode de transport autre que la conduite individuelle [9, 23, 24]. Parmi les études qui soulignent l'efficacité des mesures financières, nous trouvons certaines analyses belges relatives aux indemnités complémentaires pour l'usage du vélo et des transports en commun [12,13]. Les analyses menées au sein de l'administration fédérale belge en charge de la mobilité (officiellement dénommée le Service public fédéral (SPF) Mobilité \& Transports) insistent également sur l'efficacité des mesures financières, en particulier le remboursement partiel ou complet de l'abonnement de train ou de bus-tram-métro [25]. Si l'efficacité des mesures 
à caractère financier est fréquemment soulignée par les travaux sur les choix modaux, rien dans ces travaux ne permet toutefois de préciser l'ordre de grandeur des impacts effectifs.

Après avoir passé en revue les principaux acquis des travaux sur les pratiques modales, venons-en maintenant aux enseignements des travaux ayant cherché à quantifier l'impact de la gestion de la mobilité par les entreprises. Un recensement en a récemment été publié par Cairns et al. [24]. Il s'en dégage que l'intérêt des chercheurs s'est principalement focalisé sur des cas de bonnes pratiques qui ne sont pas nécessairement représentatifs de la diversité des situations. Selon cette littérature focalisée sur les « bonnes pratiques », les plans de mobilité d'entreprise permettent fréquemment une réduction de la conduite automobile se ventilant entre $15 \%$ et $20 \%$. Pour le contexte français, non pris en compte par Cairns et al., certains plans de mobilité d'entreprise auraient entraîné jusqu'à $10 \%$ de changements dans les modes de transport utilisés sur le trajet domicile-travail [26]. Pour Rye [7], le fait que de nombreux plans n'intègrent pas de mesures dissuasives - en particulier en matière de stationnement - tend à fortement limiter leur impact effectif sur l'autosolisme. Il en déduit que l'impact moyen doit être bien plus limité que les optimistes 15-20\% et il avance l'hypothèse d'une réduction moyenne de $6 \%$. Il souligne toutefois qu'il s'agit là d'un ordre de grandeur à considérer comme une supposition probable plutôt que comme une estimation calibrée en utilisant une méthode robuste.

La littérature dédiée aux plans de mobilité a comme point commun avec les autres recherches sur les préférences modales de conclure sur l'importance des mesures dissuasives limitant le stationnement. Cela est mis en avant tant pour la Belgique [10, 20] que pour les Pays-Bas [27, 28], le Royaume-Uni [24] ou les États-Unis [29]. Pour Rye [7], c'est précisément l'intégration de mesures dissuasives sur le stationnement qui permet de passer d'une réduction moyenne de l'autosolisme de $\pm 6 \%$ à une réduction qui approche l'ordre de grandeur des $15 \%$ typique des bonnes pratiques. À propos de l'efficacité des mesures relatives au stationnement, soulignons encore qu'elle est mise en avant pour l'ensemble des modes alternatifs à la conduite, qu'il s'agisse des mobilités douces [30], des transports collectifs [31] ou du covoiturage [32]. Tous ces travaux confirment qu'un report modal significatif nécessite tant une bonne accessibilité par les modes alternatifs qu'une politique restrictive envers la voiture [33]. En d'autres termes, il faut simultanément utiliser le « bâton » et la « carotte » dans la mise en place des politiques visant à limiter l'utilisation de l'automobile [34].

À l'issue de notre analyse de la littérature, il apparaît qu'en dépit de l'intérêt croissant de la recherche pour la gestion de la mobilité par les entreprises, les travaux actuels ne permettent pas de dégager de conclusions bien tranchées quant à l'effet réel des mesures adoptées [6]. Dans cette perspective, il nous semble que la recherche doit progresser dans deux directions à la fois complémentaires et étroitement liées. Il s'agit d'abord de considérer des panels d'entreprises représentatifs et de ne plus se limiter à des études de cas dédiées aux bonnes pratiques. En complément, il s'agit aussi de mieux préciser les interdépendances entre la gestion de la mobilité par les entreprises et les autres déterminants des pratiques modales. C'est dans cette double orientation que nous avons développé les analyses présentées ci-dessous. Pour les interdépendances entre les déterminantes des pratiques modales, ce sont les relations entre les environnements spatiaux des entreprises et les mesures adoptées que nous avons privilégiées. Pour ce qui est de la représentativité des entreprises, notre travail s'est appuyé, non pas sur des études de cas, mais sur des données représentatives de la réalité wallonne. Voyons maintenant pourquoi et comment ces données ont été collectées.

\section{Données utilisées}

\section{Les informations disponibles grâce aux diagnostics sur les déplacements domicile-travail}

En Belgique, les mesures de l'État fédéral visant à développer une politique de gestion de la mobilité par les entreprises ont intégré, par une Loi-programme datant du 8 avril 2003, l'obligation de réaliser un diagnostic relatif aux déplacements des travailleurs entre leur lieu de domicile et leur lieu de travail. Cette imposition, qui s'applique sur une base trisannuelle, concerne les unités d'établissement d'au moins 30 travailleurs qui appartiennent à des entreprises privées ou à des institutions publiques employant au moins 100 travailleurs. Les premiers diagnostics ont été réalisés en 2005, les deuxièmes en 2008 et les troisièmes en 2011. Cette réglementation vise tant à disposer d'informations sur les pratiques de mobilité des travailleurs qu'à stimuler les entreprises à instaurer des mesures susceptibles de les influencer [35].

La réalisation de ces diagnostics s'appuie sur des enquêtes qui, pour le niveau de l'établissement, permettent d'établir la part des différents modes de transport utilisés entre le lieu de domicile et le lieu de travail. Ces enquêtes sont organisées par le SPF Mobilité \& Transports. Afin de préparer cet article, nous avons utilisé une base de données de cette administration. Cette source permet d'accéder à certaines informations relatives aux enquêtes menées en 2008. Les enquêtes réalisées en 2008 sur le territoire wallon concernent 2287 unités d'établissement et 314799 salariés. Cela représente $24 \%$ de la population active occupée en Wallonie [8]. Parmi ces 2287 unités d'établissement, 
seules 1 463, comptabilisant 243044 salariés, ont pu être prises en compte par notre analyse. Pour les 824 restantes, nous ne disposions pas de l'ensemble des informations nécessaires aux traitements car les questionnaires n'ont pas été suffisamment bien complétés.

La ventilation entre modes concerne ici le déplacement « principal », c'est-à-dire celui avec lequel est parcourue la plus grande distance sur le trajet domicile-travail. Les informations disponibles ne permettent donc, ni la prise en compte des déplacements intermodaux (par exemple voiture - train), ni la prise en compte des pratiques multimodales (changements de modes entre l'aller et le retour, entre jours de la semaine, entre parties de l'année...). Concernant le mode de déplacement principal, les rubriques suivantes pouvaient être choisies : la voiture, le train, les bus-tram-métro, le transport collectif organisé par l'employeur, le vélo, les cyclomoteurs et motos, les déplacements uniquement piétons et une rubrique « autre ». Selon le SPF Mobilité et Transports, la rubrique « autre »a été choisie par certains salariés aux pratiques multimodales [25]. Concernant l'usage de la voiture, le questionnaire différencie « la voiture seul ou avec des membres de la famille » et « la voiture avec d'autres travailleurs (salariés dans la même unité d'établissement ou autre part) ». Cette différenciation permet de cerner la problématique du covoiturage mais elle ne permet malheureusement pas de différencier les rôles de conducteur et de passager.

En plus des informations sur les pratiques modales, la base de données utilisée intègre des informations sur les politiques de mobilité des entreprises. Une première information est celle du nombre de places de stationnement au sein de chaque établissement. Pour les mesures adoptées en termes de gestion de la mobilité, nous disposions du nombre de mesures par établissement pour les quatre catégories suivantes : les mesures visant à renforcer l'usage $\mathrm{du}$ vélo, les mesures visant à renforcer la pratique du covoiturage, les mesures visant à renforcer l'usage des transports en commun ainsi que les mesures « diverses ». Des mesures en faveur du vélo ont été adoptées par un nombre important d'établissements (69\%). Les principales sont la possibilité de bénéficier d'indemnités de déplacement, la présence d'un espace pour se doucher et se changer ainsi que l'aménagement d'abris couverts et sécurisés pour les vélos. Les principales mesures visant à renforcer l'usage des transports en commun sont la possibilité de bénéficier d'indemnités de déplacement, la diffusion d'informations ainsi que la mise en place d'une concertation avec les sociétés de transports en commun. Sur l'ensemble des établissements, $43 \%$ ont pris ce type de mesures en faveur des TC. Concernant le covoiturage, le questionnaire fait notamment référence à son organisation au niveau de l'établissement. Le nombre d'entreprises ayant initié ce type de mesures est assez limité (18\%). Enfin, les mesures
« diverses » les plus utilisées sont la désignation d'un coordinateur de mobilité au sein de l'établissement ainsi que l'encouragement au télétravail. Nous trouvons dans notre échantillon une part de $21 \%$ d'établissements ayant adopté ce type de mesures.

Parmi les informations disponibles grâce à la base de données du SPF Mobilité \& Transports, nous trouvons également la distance moyenne parcourue par les travailleurs lors de leurs navettes de travail. En outre, deux données portent sur l'accessibilité des établissements par les alternatives à l'automobile. Il s'agit de la présence ou de l'absence d'un arrêt de train à moins d'un kilomètre et de la présence ou de l'absence d'un arrêt de bus-tram-métro à moins de 500 mètres. Il s'agit là d'informations assez sommaires qui ne prennent en compte ni la fréquence des passages ni le nombre de lignes disponibles. Concernant la variable relative aux bus-tram-métro, ajoutons qu'elle est peu discriminante. En effet, sur les 243044 salariés considérés, seuls $12,7 \%$ sont actifs dans un établissement situé à plus de 500 mètres d'un point d'arrêt.

\section{Le profil d'accessibilité des établissements aux échelles macro et micro}

Grâce à la base de données du SPF Mobilité \& Transports, nous avons pu disposer de l'adresse postale des 1463 établissements considérés. Cette information a permis de considérer leur profil d'accessibilité à une échelle macro et à une échelle micro. Afin de préciser le profil d'accessibilité macro, nous avons utilisé une typologie des communes qui tient compte du phénomène urbain. Cela se justifie car, comme l'a bien montré J.-P. Hubert, la Belgique continue de se caractériser par des pratiques de mobilité différentes entre les zones urbaines, périurbaines et rurales [36]. Bien que l'usage de l'automobile ait fortement progressé au sein des villes, les densités plus fortes continuent d'y induire une utilisation plus importante des transports en commun et des modes doux (marche et vélo).

Afin d'établir cette typologie, nous avons choisi de différencier sept groupes de communes, comme l'illustre la carte 1 . Pour ce faire, nous avons combiné deux typologies antérieures. La première, mise au point par E. Van Hecke, rend compte de la hiérarchie urbaine [37]. Elle a permis de différencier trois premiers groupes : les grandes villes, les villes moyennes et les petites villes. Afin de classer les autres municipalités, c'est le travail d'analyse relatif aux régions urbaines [38] que nous avons utilisé, conduisant ainsi à différencier les communes d'agglomération (c'est-à-dire les communes dont plus de la moitié de la population vit dans l'agglomération morphologique d'une région urbaine), les communes des banlieues périurbaines (c'est-à-dire les couronnes périurbaines proches des agglomérations) ainsi 


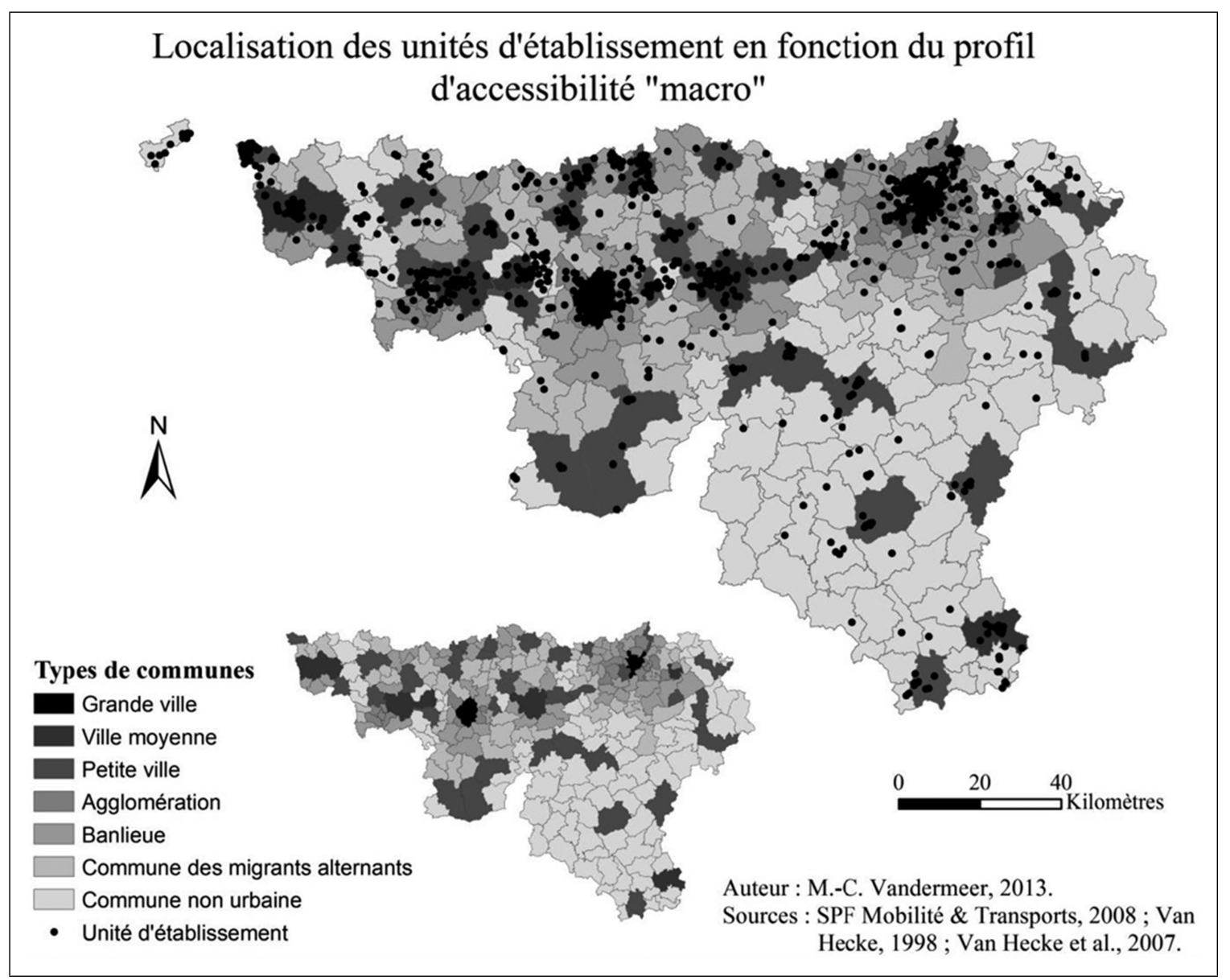

Carte 1 Localisation des unités d'établissement en fonction du profil d'accessibilité « macro »

que les communes intégrées au sein des zones résidentielles des migrants alternants (c'est-à-dire les communes périurbaines de seconde couronne). Les municipalités ne correspondant pas à un des six types prédéfinis ont été considérées comme des communes «non urbaines ».

$\mathrm{Vu}$ la superficie relativement importante des communes wallonnes - en moyenne quatre fois plus vastes que les communes françaises - les comportements de mobilité y sont spatialement très hétérogènes. Il est donc primordial de compléter la prise en compte de la localisation des établissements à l'échelle macro par leur localisation à l'échelle micro. Dans ce cadre, nous avons choisi de valoriser le travail de la CPDT (Conférence permanente du développement territorial) relatif aux cartes d'accessibilité par les alternatives à l'automobile. Ces cartes ont été développées dans les années 2000, à l'initiative de la Région wallonne, afin de constituer un outil d'aide à la décision en matière d'implantation résidentielle et socio-économique [39]. Le lecteur intéressé pourra trouver une information détaillée sur l'élaboration de cet outil d'aide à la décision chez Daxhelet et al. [40] ainsi que chez Cornet et al. [41].
Le principe d'aménagement spatial sous-jacent à l'outil des cartes d'accessibilité de la CPDT consiste en la recherche de l'adéquation entre le profil d'accessibilité des lieux et le profil de mobilité des implantations, tel que formalisé dans les années quatre-vingt par la politique néerlandaise de l'ABC [42]. L'objectif de cette cartographie est d'identifier les lieux où il existe une bonne accessibilité par les alternatives à l'automobile, afin d'y favoriser les urbanisations qui permettent de tirer le meilleur parti de ces terrains stratégiques. Pour la localisation des activités économiques, qu'il s'agisse d'entreprises privées ou d'organisations publiques, cela implique de valoriser les périmètres les plus accessibles en y concentrant des activités qui se caractérisent par des densités importantes d'emplois et/ou de visiteurs.

Les techniques géomatiques et statistiques utilisées pour préparer les cartes d'accessibilité de la CPDT ont permis d'établir des Parts modales attendues (PMA) pour les principaux modes de transport alternatifs à la voiture : le train, les bus-tram-métro et les modes doux (marche et vélo). En intégrant ces trois types d'alternatives, il devient possible, par soustraction, d'estimer la part attendue 


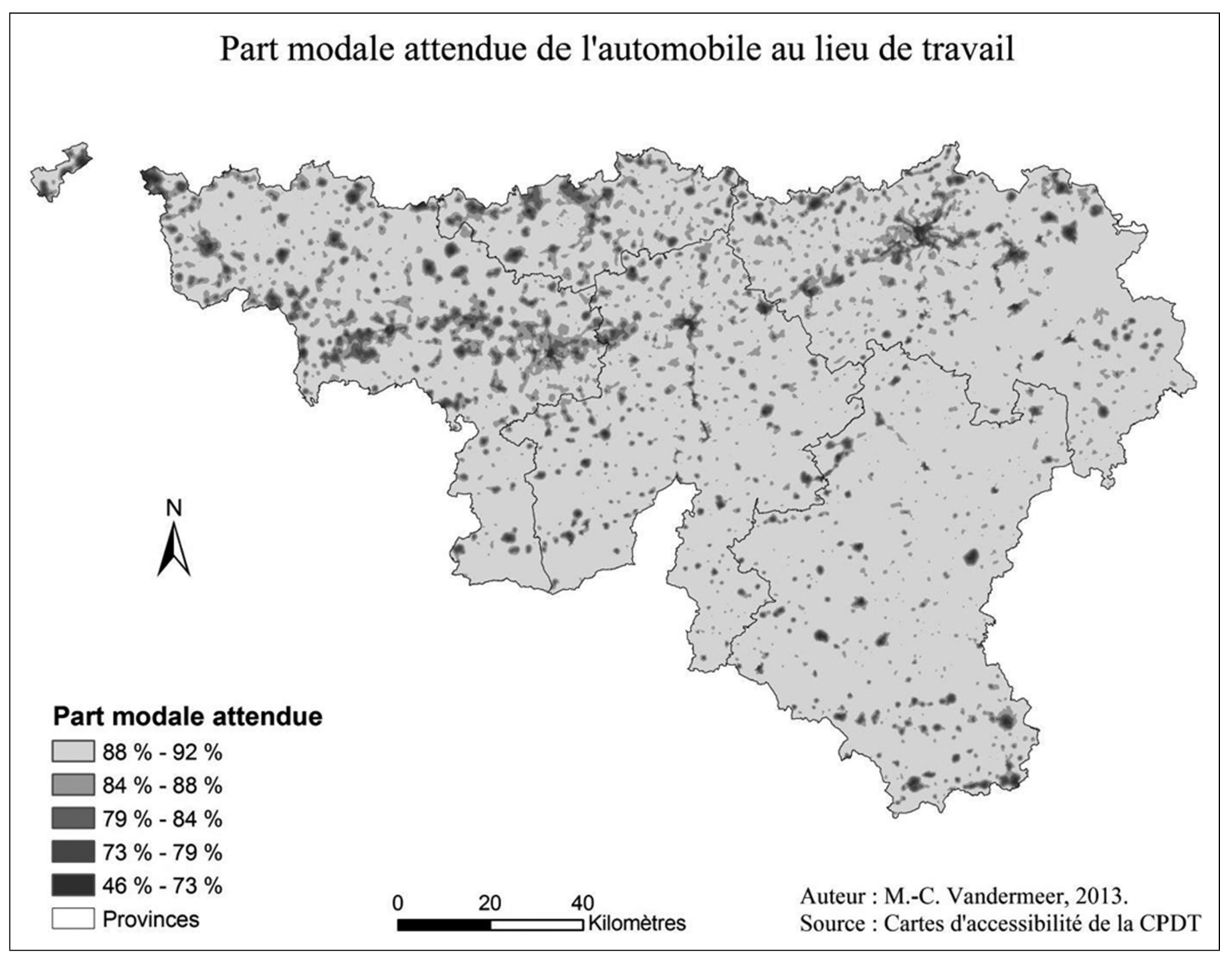

Carte 2 Part modale attendue de l'automobile au lieu de travail

pour l'automobile. Les cartes d'accessibilité renseignent les PMA tant au lieu de résidence qu'au lieu de travail, à une échelle de pixels - c'est-à-dire de carrés - de 50 mètres de côté [41]. Sans conteste, cette finesse à laquelle les cartes d'accessibilité permettent d'appréhender les comportements de mobilité est un atout fondamental de l'outil. En guise d'illustration, nous avons préparé la carte 2. Pour l'ensemble de la Wallonie, elle rend compte de la part modale attendue de l'automobile au lieu de travail.

Le territoire wallon représente approximativement 6400000 pixels. Pour chacun d'eux, les PMA ont été établies en fonction de l'offre disponible en bus et en train (sur base de la distance des pixels aux points d'arrêts et de la fréquence de passage à ces points d'arrêts) ainsi que de la densité de population dans un rayon de 1600 mètres autour du centre des pixels (afin de chiffrer le potentiel de destinations accessibles par les modes doux). Une étape importante du travail a consisté dans l'encodage de ces données primaires sur base de la situation en 2001. Ensuite, des relations mathématiques entre ces données et les PMA ont été déterminées par régression multiple, en se calibrant sur les déplacements domicile-travail observés lors du recensement de 1991. Lors de la réalisation des cartes d'accessibilité, le choix s'est porté sur ces données car les données du recensement de 2001 n'étaient pas encore encodées et disponibles.

Le tableau 1 a été préparé afin d'évaluer la robustesse de l'information disponible grâce aux cartes d'accessibilité de la CPDT. Il porte sur la comparaison entre, d'une part, les PMA au lieu de travail renseignées par cet outil et, d'autre part, les Parts modales observées (PMO) obtenues via le diagnostic sur les déplacements domicile-travail de 2008. Cette comparaison a été établie en fonction de cinq configurations de PMA pour la voiture. Comme pour l'ensemble des traitements présentés dans cet article, les moyennes reprises dans le tableau 1 ont été calculées en tenant compte du nombre de salariés au sein de chaque unité d'établissement.

Les cartes d'accessibilité permettent de considérer quatre modes de transport. L'outil renseigne directement sur les usages attendus du train, des bus-tram-métro, des modes doux (la marche et le vélo) et, indirectement, sur l'usage attendu de la voiture. En plus de ces modes, les questionnaires des diagnostics qu'impose la Loi-programme de 2003 prévoient trois autres possibilités : les transports collectifs organisés par les employeurs, les cyclomoteurs et motos 
ainsi que la rubrique « autres modes de transport». Pour l'ensemble des unités d'établissement, ces trois possibilités représentent 2,3\% des choix opérés. Afin d'établir la comparaison entre les PMA et les PMO, nous avons choisi de calculer des PMO dont le total représente $100 \%$. Nous avons pour cela reporté les parts des trois possibilités non prises en compte sur les quatre modes renseignés par les cartes d'accessibilité. Pour chaque unité d'établissement, ce report a été établi de manière proportionnelle aux parts modales initiales. Comme l'indique le tableau 1, cette manière de traiter l'information aboutit à une PMO de $86,3 \%$ pour l'automobile. Sans le report des modes ne pouvant être pris en compte, cette part serait de $84,3 \%$. Rappelons que la part modale observée de l'automobile regroupe les déplacements effectués comme conducteur et comme passager (tant avec des membres de sa famille qu'avec d'autres travailleurs).

Le tableau 1 confirme la relation entre le profil d'accessibilité micro et les comportements de mobilité. En effet, on y dégage une relation très nette entre les PMA pour la voiture et les comportements de mobilité. Plus précisément, on y observe un usage de moins en moins important de l'automobile au fur et à mesure de la progression des parts attendues pour ses alternatives. Les PMO se ventilent ainsi entre 95,2\% pour les lieux les moins accessibles par ces modes alternatifs (les lieux les plus périphériques) et $73,1 \%$ pour les lieux où cette accessibilité est la meilleure (les lieux les plus centraux). L'ANOVA-1 (analyse de la variance à un critère de classification) - calculée sur base du nombre d'établissements - se conclut par une p-value inférieure à 0,0001 , aussi bien pour la PMO de la voiture que pour les PMO des trois autres modes de transport. Les comportements de mobilité observés sont donc bien statistiquement dépendants des profils d'accessibilité micro que renseignent les cartes d'accessibilité de la CPDT.
Concernant l'usage de la voiture, le tableau 1 indique que l'écart entre les PMA et les PMO est systématiquement négatif. Nous en déduisons que les cartes d'accessibilité surestiment l'usage des modes alternatifs. Cela peut résulter d'une mauvaise calibration des modèles mathématiques mais aussi de l'ancienneté des données de référence qui, rappelons-le, proviennent du recensement de 1991. En effet, la sous-estimation de la part de la voiture peut être provoquée par le renforcement de l'usage automobile depuis cette date [43]. Le tableau 1 indique également que, pour la voiture, la différence entre les PMA et les PMO est particulièrement importante au sein des périmètres les plus accessibles par les modes alternatifs. Cette situation tient vraisemblablement au fait que les modèles utilisés pour les cartes d'accessibilité surestiment la part des bus-tram-métro et des modes doux là où ces modes bénéficient de la meilleure accessibilité, comme en a témoigné l'analyse spatiale des écarts - des résidus - à ces modèles [41]. Observons encore que la part du train est inférieure à celle attendue dans les lieux peu accessibles par les modes alternatifs à la voiture. Par contre, dans les lieux les plus accessibles, la part du train est fortement sous-estimée.

Les résultats rassemblés dans le tableau 1 vérifient que les cartes d'accessibilité demeurent d'actualité pour rendre compte de la géographie fine du profil d'accessibilité des lieux. Bien que l'outil ait été mis en place voici une dizaine d'années, l'organisation des réseaux de transport wallons n'a pas connu de changements notables depuis lors. Par exemple, les lieux qui étaient accessibles par les alternatives à l'automobile en 2008 étaient déjà des lieux centraux au début de la décennie 2000. Concernant l'utilisation des données du recensement de 1991 pour le calibrage des PMA, il est raisonnable de considérer que la manière dont la structure de l'espace détermine les comportements de mobilité et les choix modaux n'a pas été fondamentalement

\begin{tabular}{|c|c|c|c|c|c|c|c|c|c|c|c|c|}
\hline \multirow[t]{2}{*}{ PMA voiture } & \multicolumn{3}{|c|}{ Voiture } & \multicolumn{3}{|l|}{ Train } & \multicolumn{3}{|c|}{ Bus-tram-métro } & \multicolumn{3}{|c|}{ Modes doux } \\
\hline & PMA & PMO & Écart & PMA & PMO & Ecart & PMA & PMO & Ecart & PMA & PMO & Ecart \\
\hline De $88 \%$ à $92 \%$ & 89,8 & 95,2 & $-5,4$ & 1,9 & 1,3 & 0,6 & 3,3 & 1,9 & 1,4 & 5,0 & 1,6 & 3,4 \\
\hline De $84 \%$ à $88 \%$ & 86,1 & 91,1 & $-5,0$ & 2,0 & 1,9 & 0,1 & 4,0 & 3,7 & 0,3 & 7,9 & 3,3 & 4,6 \\
\hline De $79 \%$ à $84 \%$ & 81,8 & 86,8 & $-5,0$ & 2,3 & 3,1 & $-0,8$ & 4,4 & 4,4 & 0,0 & 11,5 & 5,7 & 5,8 \\
\hline De $73 \%$ à $79 \%$ & 76,4 & 81,7 & $-5,3$ & 3,6 & 6,1 & $-2,5$ & 5,6 & 4,4 & 1,2 & 14,4 & 7,8 & 6,6 \\
\hline De $46 \%$ à $73 \%$ & 65,0 & 73,1 & $-8,1$ & 10,0 & 14,0 & $-4,0$ & 10,5 & 5,5 & 5,0 & 14,5 & 7,4 & 7,1 \\
\hline TOTAL & 80,6 & 86,3 & $-5,7$ & 3,7 & 4,9 & $-1,2$ & 5,3 & 3,8 & 1,5 & 10,3 & 5,0 & 5,3 \\
\hline p-value (ANOVA-1) & \multicolumn{3}{|c|}{$<0,0001$} & \multicolumn{3}{|c|}{$<0,0001$} & \multicolumn{3}{|c|}{$<0,0001$} & \multicolumn{3}{|c|}{$<0,0001$} \\
\hline
\end{tabular}


bouleversée depuis lors. Pour autant, comme nous venons de l'observer, il faut être attentif à la progression généralisée de l'usage automobile.

\section{La méthode adoptée en vue de maîtriser les effets de composition}

Nous l'avons mentionné ci-dessus, les mesures adoptées par les entreprises se rapportent à quatre grandes catégories : les mesures visant à renforcer l'usage du vélo, les mesures visant à renforcer la pratique du covoiturage, les mesures visant à renforcer l'usage des transports en commun ainsi que les mesures qualifiées de «diverses ». Rappelons que ces mesures « diverses » font principalement référence à la désignation d'un coordinateur de mobilité au sein de l'établissement ainsi qu'à l'encouragement au télétravail.

Le tableau 2 permet une première analyse de l'impact des mesures visant à encourager l'usage des transports en commun. Selon nos traitements, il s'agit là du type de mesures qui détermine les modifications les plus notables. Rappelons que parmi les mesures en faveur des transports en commun, le remboursement par l'employeur des abonnements de train et de bus-tram-métro est considéré comme une pratique particulièrement efficace. Le tableau 2 indique une différence du simple au double dans le recours aux transports collectifs entre les entreprises qui n'ont pas pris de mesures et les entreprises qui en ont adopté (de $5,8 \%$ à $11,3 \%$ ). L'effet est particulièrement notable pour le train qui fait plus que doubler sa part modale (de 2,8 \% à $6,8 \%$ ). En parallèle, notons une différence de $6,8 \%$ (de $87,7 \%$ à $80,9 \%$ ) quant à l'utilisation de la voiture entre les entreprises qui n'ont pas pris de mesures et les entreprises qui en ont adoptées. Sur un plan statistique, la variation des pourcentages est dépendante de la prise - ou non - de mesures en faveur des transports en commun. En effet, l'ANOVA-1 se conclut par une p-value inférieure à 0,0001 , aussi bien pour la voiture que pour le train et les bus-tram-métro. Précisons que si les parts modales ont été établies sur base du nombre de salariés (n), les p-values ont été calculées en considérant le nombre d'établissements (n').

Il convient d'être très prudent vis-à-vis des conclusions à retirer du tableau 2. En effet, les résultats qui y sont rassemblés sont partiellement induits par les mesures considérées, mais ils sont également influencés par le fait que les entreprises ayant initié des mesures se situent généralement dans des contextes favorables pour le faire. Par exemple, les établissements situés à moins d'un kilomètre d'un arrêt de train ont été particulièrement nombreux à adopter des mesures visant à renforcer l'usage des transports en commun $(55 \%$ contre $37 \%$ pour les établissements situés à plus d'un kilomètre d'un arrêt de train). Les interdépendances entre la gestion de la mobilité par les entreprises et d'autres déterminants des pratiques modales sont donc à l'origine d'effets de composition qu'il convient de maîtriser pour travailler " toutes choses égales par ailleurs ».

Afin de contrôler des effets de composition, il est généralement fait appel à la statistique multivariée et à l'outil statistique de la régression multiple. Au final, nous n'avons pas procédé de la sorte car les modèles économétriques calibrés sur nos données ne se sont pas révélés très riches d'enseignement. Le caractère peu concluant de l'approche économétrique s'explique par la complexité des relations à modéliser. Cela tient d'abord à des phénomènes d'interactions entre variables. En effet, certaines variables - par exemple l'accessibilité micro peuvent simultanément influencer les parts modales et l'efficacité des mesures visant à impacter les parts modales. Une complexité supplémentaire résulte du fait que les interactions ne sont pas nécessairement modélisables par des fonctions monotones. Par exemple, comme nous le détaillons ci-dessous, il existe une relation en $U$ inversé entre l'accessibilité micro et l'efficacité des mesures en faveur des transports en commun. En effet, ces mesures sont plus efficaces au sein des établissements à l'accessibilité intermédiaire qu'au sein des établissements à la localisation très centrale ou très périphérique.

Tableau 2 Parts modales moyennes observées en fonction des mesures prises en faveur des transports en commun

\begin{tabular}{|c|c|c|c|c|}
\hline & Voiture & Train & Bus-tram-métro & $\begin{array}{l}\text { Transports } \\
\text { en commun }\end{array}$ \\
\hline Pas de mesures en faveur des T. C. $(n=121601 ; n ’=827)$ & $87,7 \%$ & $2,8 \%$ & $3,0 \%$ & $5,8 \%$ \\
\hline Au moins une mesure en faveur des T.C. $\left(n=121443 ; n^{\prime}=636\right)$ & $80,9 \%$ & $6,8 \%$ & $4,5 \%$ & $11,3 \%$ \\
\hline Ensemble des entreprises $\left(n=243044 ; n^{\prime}=1463\right)$ & $84,3 \%$ & $4,8 \%$ & $3,8 \%$ & $8,6 \%$ \\
\hline p-value (ANOVA-1) & $<0,0001$ & $<0,0001$ & $<0,0001$ & $<0,0001$ \\
\hline
\end{tabular}


Face à l'impossibilité de modéliser des relations aussi complexes, la méthode alternative a consisté à calculer des sommes pondérées en ayant préalablement segmenté l'échantillon sur base des facteurs qui déterminent les choix modaux. Ces sommes ont été pondérées en tenant compte du nombre de salariés présents dans chaque établissement. Leur calcul a différencié, d'une part, les établissements n'ayant pas adopté de mesures et, d'autre part, les établissements en ayant adopté au moins une. Cela donne des simulations qui permettent d'opposer deux configurations théoriques. La première est celle qu'il aurait été probable d'observer si aucune entreprise n'avait adopté de mesures (configuration $A$ dans les tableaux ci-dessous). La seconde, à l'opposé, renvoie à une situation où au moins une mesure aurait été adoptée par l'ensemble des établissements (configuration C). Les deux configurations théoriques $\mathrm{A}$ et $\mathrm{C}$ sont à comparer à la situation réellement observée (configuration $B)$. La différence entre les configurations $\mathrm{A}$ et $\mathrm{C}$ est à interpréter de deux manières. Au niveau agrégé, elle donne une estimation de l'effet maximal susceptible d'être atteint si l'ensemble des entreprises adoptait au moins une mesure. À l'échelle des unités d'établissement, elle donne une estimation de l'impact moyen accompagnant la prise de mesures.

\section{Présentation et discussion des résultats}

\section{L'impact des mesures visant à renforcer l'usage des transports en commun (TC)}

Nous reprenons dans ce chapitre les principaux résultats d'une analyse ayant testé l'effet de différentes variables sur, d'une part, l'utilisation des TC et sur, d'autre part, l'efficacité des mesures adoptées par les entreprises afin de renforcer l'utilisation des TC. Parmi les variables testées, les résultats les plus significatifs ont été obtenus pour la distance moyenne parcourue par les travailleurs et pour les profils d'accessibilité. Ce sont donc ces variables qui, comme nous le détaillons ci-dessous, ont été utilisées afin de quantifier l'impact des mesures visant à accroître la part modale des TC. Par contre, les résultats ont été moins probants - tant concernant l'influence sur l'utilisation des TC que sur l'influence des mesures visant à la renforcer - pour les variables du nombre de salariés, du secteur d'activité et du ratio entre le nombre d'emplois et le nombre de places de stationnement disponibles dans l'établissement.

Concernant la variable du nombre de salariés, soulignons qu'elle est discriminante sur la prise de mesures mais qu'elle n'est pas corrélée à la part modale des TC. En d'autres termes, la taille des établissements semble influencer la prise de mesures mais elle ne semble pas influencer la fréquentation des TC. Concernant la variable du secteur d'activité, clarifions la problématique en faisant référence à une analyse antérieure établie à partir des données utilisées pour cet article [44]. Cette analyse a vérifié qu'il existe de fortes différences entre secteurs quant aux ventilations modales. Par exemple, l'usage de l'automobile est bien plus important dans le commerce de gros qu'au sein de l'enseignement ou des administrations publiques. Toutefois, des différences entre secteurs ne sont plus observées lorsque les comparaisons sont établies entre des entreprises situées dans les mêmes types d'accessibilité. Cela indique que les pratiques différenciées entre secteurs tiennent moins à des effets intrinsèques qu'à des localisations différenciées.

Attardons-nous maintenant sur le sujet du stationnement et sur le caractère non discriminant de la variable du ratio entre le nombre d'emplois et le nombre de places de stationnement. Nous l'avons rappelé ci-dessus, la restriction en offre de stationnement est reconnue comme une mesure particulièrement efficace par les recherches sur le report modal. En milieu urbain, limiter le stationnement est crucial pour mener une politique restrictive envers l'automobile. Dans cette perspective, ne pas observer de relations entre la disponibilité en places de stationnement dans l'établissement et l'usage des transports collectifs tient sans doute aux caractéristiques du contexte wallon où, d'une part, l'emploi s'est fortement périurbanisé [45] et où, d'autre part, les villes et communes ne mènent pas de politiques très restrictives vis-à-vis du stationnement [46]. En conséquence, au sein de notre population d'entreprises, les travailleurs peuvent assez facilement stationner dans l'espace public et les difficultés de parcage ne concernent sans doute qu'un nombre très limité d'établissements.

Le tableau 3 rend compte d'une simulation établie en fonction du facteur de la distance moyenne parcourue par les travailleurs lors de leurs déplacements domicile-travail. Cet indicateur, disponible uniquement à l'échelle de l'établissement, est extrait de la base de données du SPF Mobilité \& Transports commentée ci-dessus. Puisqu'il s'agit de la première simulation, détaillons le mode opératoire qui permet d'obtenir les moyennes pondérées. Par exemple, s'agissant de la valeur de $88,0 \%$ relative à la configuration A (configuration probable si aucune des entreprises de l'échantillon n'avait adopté de mesures), ce résultat a été obtenu en multipliant les différentes valeurs renseignées pour cette configuration $(80,4 \%-88,3 \%-$ $90,2 \%-71,4 \%$ p par le nombre de travailleurs appartenant à chaque catégorie respective (20 944-110 048 - 106094 - 5 958). Pour aboutir à l'évaluation finale, le résultat de cette somme a été divisé par le nombre total de travailleurs (243 044). À propos du contenu du tableau 3, signalons encore qu'il précise la part des travailleurs actifs dans un établissement ayant adopté au moins une mesure (41\% par exemple pour les établissements où la distance moyenne des navettes est inférieure à $10 \mathrm{~km}$ ). 
Tableau 3 Quantification de l'effet des mesures en faveur des transports en commun en fonction de la distance domicile-travail moyenne

\begin{tabular}{|c|c|c|c|c|c|c|}
\hline & \multicolumn{3}{|c|}{ Part modale moyenne de la voiture } & \multicolumn{2}{|c|}{ Différence entre les config. } & \multirow[t]{2}{*}{ p-value } \\
\hline & Config. A & Config. B & Config. $\mathrm{C}$ & A et $B$ & A et $\mathbf{C}$ & \\
\hline$<10 \mathrm{~km}\left(\mathrm{n}=20\right.$ 944/MES TC $\left.=41 \% ; \mathrm{n}^{\prime}=198\right)$ & $80,4 \%$ & $79,8 \%$ & $78,9 \%$ & $0,6 \%$ & $1,5 \%$ & 0,1311 \\
\hline $10-20 \mathrm{~km}\left(\mathrm{n}=110048 / \mathrm{MES}\right.$ TC $\left.=42 \% ; \mathrm{n}^{\prime}=659\right)$ & $88,3 \%$ & $86,6 \%$ & $84,3 \%$ & $1,7 \%$ & $4,0 \%$ & 0,0001 \\
\hline $20-45 \mathrm{~km}\left(\mathrm{n}=106094 / \mathrm{MES}\right.$ TC $\left.=60 \% ; \mathrm{n}^{\prime}=561\right)$ & $90,2 \%$ & $83,5 \%$ & $79,1 \%$ & $6,7 \%$ & $11,1 \%$ & 0,0001 \\
\hline$>45 \mathrm{~km}\left(\mathrm{n}=5958 / \mathrm{MES}\right.$ TC $\left.=47 \% ; \mathrm{n}^{\prime}=45\right)$ & $71,4 \%$ & $70,7 \%$ & $70,0 \%$ & $0,7 \%$ & $1,4 \%$ & 0,5445 \\
\hline $\begin{array}{l}\text { Moyenne pondérée }(n=243044 / \text { MES TC }=50 \% \text {; } \\
\left.n^{\prime}=1463\right)\end{array}$ & $88,0 \%$ & $84,3 \%$ & $81,2 \%$ & $3,7 \%$ & $6,8 \%$ & 0,0001 \\
\hline
\end{tabular}

Concernant l'effet des mesures sur la part modale de l'automobile, les simulations rassemblées dans le tableau 3 indiquent qu'elles ont permis une réduction de 3,7 \%. Une réduction supplémentaire de $3,1 \%$ aurait pu être concrétisée si l'ensemble des unités d'établissement avait développé une politique active. Nous aboutissons donc à un effet théorique maximal de $6,8 \%$, équivalent à l'effet mesuré sans l'application de la méthode des sommes pondérées.

Un résultat à retirer du tableau 3 est la faiblesse de la part modale de la voiture pour les distances moyennes supérieures à 45 kilomètres (approximativement $70 \%$ ). Les établissements où de nombreux travailleurs doivent se déplacer sur de longues distances se caractérisent donc par un usage limité de l'automobile. Ce résultat est cohérent avec de nombreux travaux ayant montré que l'usage du train augmente avec l'allongement de la navette domicile-travail $[20,43]$.

Le tableau 3 indique également que les mesures en faveur des transports en commun influencent significativement la part modale de la voiture pour des distances moyennes de déplacement comprises entre 10 et 45 kilomètres. Par contre, pour les distances inférieures à 10 kilomètres et supérieures à 45 kilomètres, l'impact des mesures n'est pas statistiquement significatif. Le tableau 3 montre aussi que l'effet des mesures en faveur des transports en commun progresse avec la distance avant d'atteindre un optimum qui se situe entre 20 et 45 kilomètres. Ces résultats peuvent s'interpréter en considérant une relation croissante et ensuite décroissante ( $\mathrm{U}$ inversé) entre la distance domicile-travail et l'efficacité des mesures en faveur des transports en commun (en particulier le remboursement de l'abonnement de train). Dans une première tranche de distances, cette mesure fait basculer de plus en plus de choix modaux. Ensuite, au-delà d'une distance seuil, elle devient moins efficace car de nombreux navetteurs abandonnent la voiture même s'ils ne bénéficient pas d'un défraiement.

Pour le tableau 4, la simulation a été établie en fonction de la PMA pour l'automobile, c'est-à-dire en fonction de l'accessibilité par les modes de transport alternatifs (profil d'accessibilité micro). L'effet actuel est cette fois évalué à une réduction de $2,8 \%$ pour la part de l'automobile, avec un effet potentiel supplémentaire de $2,7 \%$. Nous aboutissons ici à un effet théorique maximal de 5,5\%, inférieur de 1,3\% par rapport à l'effet mesuré sans l'application de la méthode des sommes pondérées. Afin de réduire davantage cet effet théorique maximal, nous avons testé d'autres segmentations en conjuguant la PMA pour l'automobile à d'autres facteurs explicatifs des choix modaux. Le seul résultat significatif est apparu en utilisant le profil d'accessibilité macro. Lorsque les deux profils sont conjugués, l'effet actuel est réduit à $2,3 \%$ et l'effet théorique maximal à $5,1 \%$. Rappelons que cet effet théorique maximal peut être appréhendé comme l'impact moyen accompagnant la prise de mesures à l'intérieur d'une unité d'établissement.

Afin d'expliquer pourquoi la segmentation en fonction des profils d'accessibilité macro et micro donne les résultats les plus robustes, il faut considérer que les choix de localisation des établissements interagissent avec de nombreux facteurs qui déterminent également les comportements de mobilité des travailleurs. En tenant compte de la localisation, nous bénéficions donc en réalité d'une information très riche sur les caractéristiques des entreprises. Au final, il apparaît que le calcul de simulations sur base des profils d'accessibilité micro et macro est un moyen efficace pour contrôler la complexité des effets de composition. Il est toutefois vraisemblable que cette approche ne permette pas une maîtrise absolue de ces effets. En conséquence, nos résultats se caractérisent probablement par une légère surestimation des effets réels. 


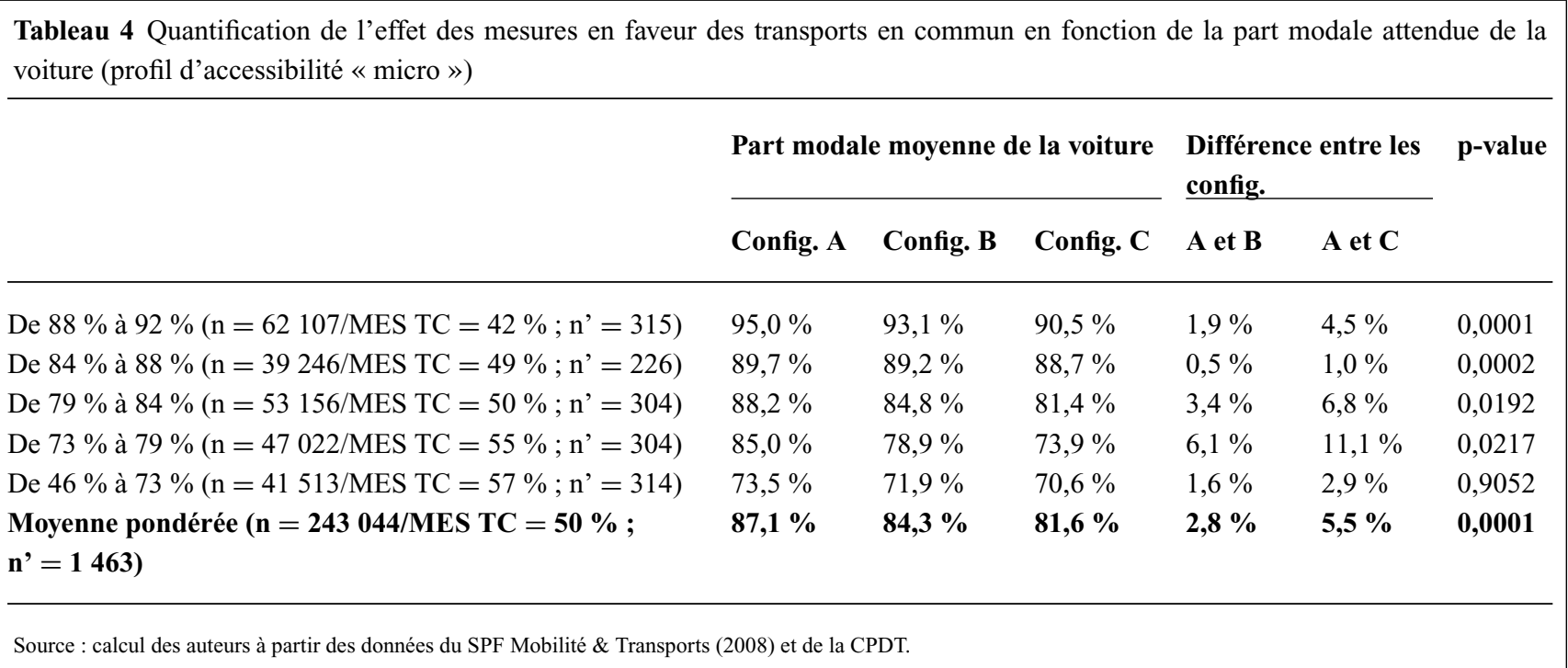

Détailler le tableau 4 permet de développer l'analyse sur les relations entre l'efficacité des mesures et la localisation des entreprises en termes d'accessibilité par les alternatives à l'automobile. Il ressort d'abord de ce tableau que la prise de mesures progresse avec l'accessibilité : $57 \%$ des travailleurs des espaces les plus centraux sont concernés par des mesures alors qu'ils ne sont plus que $42 \%$ dans les espaces les plus périphériques. Il ressort ensuite du tableau 4 que, contrairement à notre hypothèse de travail, il n'existe pas de progression automatique de l'efficacité des mesures avec l'amélioration de l'accessibilité par les modes de transport alternatifs. Comme nous l'avons mentionné ci-dessus, nous constatons une relation en U inversé entre l'accessibilité micro et l'efficacité des mesures en faveur des transports en commun. Afin d'expliquer ce résultat contreintuitif, il faut d'abord faire référence à l'influence d'effets de composition, et ce en particulier pour la classe de PMA de la voiture comprise entre $88 \%$ et $92 \%$. Dans cette catégorie, le profil des établissements qui ont adopté des mesures est très différent du profil des établissements qui n'en ont pas adopté. Des différences importantes concernent le profil d'accessibilité macro. En effet, les établissements ayant pris des mesures sont beaucoup plus nombreux que ceux n'en ayant pas pris à se situer dans une ville (70 \% contre $40 \%)$. Pour la classe de PMA de la voiture comprise entre $88 \%$ et $92 \%$, des mesures en faveur des transports collectifs ne semblent donc avoir été prises qu'au sein des établissements bénéficiant d'une desserte minimale. À l'inverse, les établissements se caractérisant par une desserte inexistante n'ont pas jugé opportun d'initier une politique active.

Intéressons-nous maintenant à la situation des établissements qui bénéficient de la meilleure accessibilité par les alternatives à l'automobile (PMA de $46 \%$ à $73 \%$ ).
Dans ces localisations, qui correspondent généralement aux centres-villes, la prise de mesures en faveur des transports en commun est associée à une réduction très faible de la part de l'automobile (effet réel de 1,6\% et effet potentiel maximal de $2,9 \%$ ). La variation de la part modale est d'ailleurs statistiquement non significative (p-value de 0,9 ). L'analyse détaillée des données montre que la divergence entre ce résultat et les résultats des autres classes d'accessibilité tient à la problématique des modes doux. On trouve ici une situation où les entreprises ayant adopté des mesures en faveur des transports en commun se caractérisent par une part de déplacements à pied ou à vélo plutôt faible (6,4 \% contre $8,4 \%$ pour les entreprises n'ayant pas pris de mesures). Pour les établissements qui bénéficient des meilleures accessibilités par les alternatives à l'automobile, le report modal vers les TC engendré par la prise de mesures semble donc être le fait de nombreux anciens usagers des modes doux.

Pour expliquer la faiblesse du report modal depuis la voiture vers les transports collectifs dans cette catégorie aux PMA de la voiture les plus faibles, observons que la qualité de la desserte y représente déjà un incitatif important à leur utilisation. Ils y sont d'ailleurs utilisés par un nombre relativement élevé de salariés. Par exemple, l'utilisation de l'automobile pour les établissements n'ayant pas adopté de mesures $(73,5 \%)$ y est inférieure à l'utilisation de l'automobile pour les établissements moins accessibles et ayant adopté des mesures (à partir de 73,9\%). Le fait que ce contexte spatial ne soit pas favorable au report vers les TC semble indiquer qu'en comparaison de l'excellente desserte, les avantages supplémentaires liés aux mesures sont négligeables - en particulier le remboursement des abonnements. Les salariés qui n'utilisent pas les transports collectifs 
alors qu'ils travaillent dans un lieu très accessible semblent donc se caractériser par des comportements « inflexibles » que l'argument supplémentaire du remboursement d'un abonnement ne modifie pas.

Les facteurs explicatifs de ces comportements apparemment inflexibles sont probablement multiples. Ils peuvent être d'ordres psychologiques ou symboliques (par exemple une aversion pour les transports collectifs) [47]. Ils peuvent aussi être liés à la possession d'une voiture de société, dont on a rappelé qu'elle peut justifier la conduite en toutes circonstances. La problématique du chaînage des déplacements peut également être à l'origine des comportements observés, par exemple par l'obligation d'utiliser la voiture pour rejoindre d'autres destinations moins centrales [48, 49]. Afin d'expliquer la faiblesse du report modal depuis la voiture vers les TC au sein des centres-villes wallons, il faut également revenir sur la problématique du stationnement. En milieu urbain, limiter le stationnement est crucial s'il s'agit de mener une politique restrictive envers l'automobile. Or, comme nous l'avons relevé ci-dessus, les politiques de stationnement des villes et communes wallonnes ne sont pas très restrictives. En conséquence, même en centre-ville, le problème du stationnement ne se pose réellement que pour un nombre limité d'établissements.

En synthèse, la prise en compte des relations entre l'efficacité des mesures et l'accessibilité par les alternatives à l'automobile conduit à différencier trois configurations spatiales. Une première correspond aux lieux les plus périphériques, où la desserte par transports en commun est quasi inexistante. Les entreprises de cette configuration sont peu enclines à développer des mesures. Une deuxième configuration spatiale est celle des centres-villes. Nous sommes ici dans un environnement qui, en Wallonie, n'est pas suffisamment défavorable à la voiture pour y limiter son usage. Il en résulte que la prise de mesures en faveur des transports en commun s'accompagne d'une substitution aux modes doux plutôt qu'à la voiture. Enfin, nous trouvons les localisations intermédiaires. Elles correspondent à des lieux situés en dehors des centres, où l'offre en transports collectifs est peu développée mais existante. Dans les faits, ce contexte spatial est le plus favorable à la prise de mesures. À la différence des centres-villes, un nombre significatif de salariés qui n'y sont pas incités par la seule qualité de la desserte se décide à abandonner la voiture lorsque les établissements mettent en œuvre des mesures qui les y encouragent. Dans ce type d'espaces, le remboursement de l'abonnement peut donc faire basculer un nombre assez élevé d'automobilistes du côté des usagers des transports collectifs.

\section{L'impact des mesures « diverses » et des mesures visant à renforcer les pratiques du vélo et du covoiturage}

Le tableau 5 porte sur les parts modales en fonction de la prise de mesures " diverses ». Il indique que ces mesures sont associées à une réduction de $1,9 \%$ de l'usage de la voiture, variation statistiquement non significative. Par contre, la variation de parts modales du train $(+2,5 \%)$ est statistiquement dépendante de l'adoption de mesures diverses. Il semblerait donc que la prise de telles mesures entraîne un léger report modal - depuis la voiture mais également depuis les modes doux - vers les transports en commun. De nouveau, il s'agit d'être prudent avec ce résultat qui, en réalité, résulte d'effets de composition liés aux mesures en faveur des transports en commun. En effet, les entreprises ayant adopté des mesures « diverses » ont aussi très fréquemment adopté des mesures relatives aux transports en commun. Lorsque cet effet de composition est contrôlé par moyenne pondérée, aucun impact significatif ne peut plus être isolé. Concernant les mesures " diverses », il faut se rappeler que les actions les plus utilisées sont la désignation d'un coordinateur de mobilité et l'encouragement au télétravail. Il n'est donc pas étonnant que notre approche ne puisse vérifier l'impact effectif de ces mesures. Afin d'approfondir leur évaluation, il faudrait travailler sur la problématique du télétravail, ce qui n'est malheureusement pas réalisable avec les données disponibles.

\begin{tabular}{|c|c|c|c|c|}
\hline & Voiture & Train & Bus-tram-métro & Modes doux \\
\hline Entreprises n'ayant pas adopté de mesures $\left(\mathrm{n}=166749 ; \mathrm{n}^{\prime}=1151\right)$ & $84,9 \%$ & $4,0 \%$ & $3,4 \%$ & $5,1 \%$ \\
\hline Entreprises ayant adopté au moins une mesure $(n=76295 ; n ’=312)$ & $83,0 \%$ & $6,5 \%$ & $4,5 \%$ & $4,2 \%$ \\
\hline Ensemble des entreprises $\left(n=243044 ; n^{\prime}=1463\right)$ & $84,3 \%$ & $4,8 \%$ & $3,8 \%$ & $4,8 \%$ \\
\hline p-value & 0,2005 & 0,0001 & 0,2047 & 0,0050 \\
\hline
\end{tabular}


À l'instar des mesures « diverses », nos traitements ne permettent pas non plus de vérifier l'hypothèse selon laquelle les mesures visant à favoriser la pratique du vélo influencent significativement l'utilisation de l'automobile. Comme l'illustre le tableau 6, la part modale de la voiture est de $84,2 \%$ pour les entreprises ayant adopté au moins une mesure en faveur de la pratique cycliste et de $84,7 \%$ pour les entreprises n'ayant rien initié en la matière. Il existe donc une différence de $0,5 \%$. Toutefois, cette différence n'est pas statistiquement significative. De plus, elle ne se confirme pas lorsque l'échantillon est segmenté sur base des facteurs qui déterminent les choix modaux (notamment lorsque les profils d'accessibilité macro et micro sont pris en compte).

Concernant l'impact des mesures en faveur du vélo, nos résultats divergent des conclusions de Vanoutrive et al. [50]. En effet, selon Vanoutrive et ses collègues, les mesures financières prises par les entreprises belges afin d'encourager l'usage du vélo seraient relativement efficaces. Cette divergence tient sans doute à l'usage très différencié du vélo au sein des trois régions belges : le vélo représente près de $13 \%$ des parts modales en Flandre mais moins de $2 \%$ en Wallonie et à Bruxelles [25]. Le contexte favorable de la Flandre pour le vélo s'explique par l'entremêlement de raisons tant topographiques que culturelles et infrastructurelles $[1,50,51]$. De telles différences de contexte signifient que les mesures en faveur des deux-roues - en particulier les mesures financières - trouvent un terreau bien plus favorable dans le nord du pays. Face à ce constat, remémorons-nous les conclusions de Pucher et al. quant à la nécessité d'intégrer les mesures en faveur du vélo dans un ensemble qui les articule à la planification des infrastructures et à l'aménagement du territoire [19].

Les données rassemblées dans le tableau 6 indiquent également que les mesures en faveur du vélo s'accompagnent bien d'une évolution significative de son utilisation. Cette évolution est ici statistiquement significative et, en outre, non dépendante d'effets de composition. Afin d'expliquer cette apparente contradiction avec les conclusions relatives à l'usage de l'automobile, nous pouvons avancer l'hypothèse qu'encourager la pratique cycliste des salariés wallons se traduit par un report modal depuis la marche. Sur base du tableau 6 , la progression de $0,8 \%$ de l'utilisation du vélo induit par la prise de mesures s'accompagne d'une réduction de $0,4 \%$ de la marche (la part des modes doux ne progresse que de $0,4 \%$ ).

Nous l'avons précisé ci-dessus, les données disponibles sur les modes de transport différencient deux usages de la voiture : « la voiture seul ou avec des membres de la famille » et « la voiture avec d'autres travailleurs (salariés dans la même unité d'établissement ou autre part) ». Cette différenciation permet de cerner la problématique du covoiturage mais elle présente malheureusement le désavantage de ne pas différencier les conducteurs des passagers.

Selon nos données, la pratique du covoiturage concerne $4,8 \%$ des salariés. Nous pouvons en déduire que la part du covoiturage en tant que passager représente un minimum de $2,4 \%$ (taux d'occupation de 2 personnes par véhicule) et un maximum de 3,8\% (taux d'occupation de 5 personnes par véhicule). La pratique du covoiturage, qui n'est guère plus répandue en Flandre ou à Bruxelles [25], peine donc à convaincre les salariés belges. Cela tient vraisemblablement à l'atteinte à l'autonomie qu'elle entraîne, atteinte d'autant plus importante qu'elle s'inscrit dans des contextes professionnels où les horaires sont de plus en plus variables et aléatoires. En outre, les déplacements domicile-travail sont très fréquemment intégrés dans des chaînes de déplacement complexes, où les lieux d'activité et de résidence ne représentent pas les seules destinations [43].

Corréler la part modale du covoiturage aux principales caractéristiques des établissements a permis d'identifier des associations significatives avec le nombre de mesures adoptées afin de renforcer cette pratique ainsi qu'avec le nombre de salariés (rappelons que le nombre de salariés n'est par contre pas corrélé à la part des TC). Les mesures en faveur du covoiturage semblent donc réellement efficaces. La progression renseignée par le tableau 7 est de 5,7\%: de $3,6 \%$ pour les entreprises n'ayant pas adopté de mesures à $9,3 \%$ pour les entreprises en ayant adopté au moins une.

Lorsque l'effet de composition lié à la taille est pris en compte (la prise de mesures est plus efficace dans les

Tableau 6 Parts modales moyennes du vélo, des modes doux et de la voiture en fonction des mesures prises en faveur du vélo

\begin{tabular}{|c|c|c|c|}
\hline & Vélo & Modes doux & Voiture \\
\hline Entreprises n'ayant pas adopté de mesures $(n=63123 ; n '=458)$ & $1,0 \%$ & $4,5 \%$ & $84,7 \%$ \\
\hline Entreprises ayant adopté au moins une mesure $\left(\mathrm{n}=179921 ; \mathrm{n}{ }^{\prime}=1005\right)$ & $1,8 \%$ & $4,9 \%$ & $84,2 \%$ \\
\hline Ensemble des entreprises $\left(n=243044 ; n^{\prime}=1463\right)$ & $1,6 \%$ & $4,8 \%$ & $84,3 \%$ \\
\hline p-value & 0,0001 & 0,7914 & 0,4590 \\
\hline
\end{tabular}




\begin{tabular}{|c|c|c|c|}
\hline & Covoiturage & $\begin{array}{l}\text { Voiture (seul ou } \\
\text { en famille) }\end{array}$ & $\begin{array}{l}\text { Voiture (seul, en } \\
\text { famille et covoiturage) }\end{array}$ \\
\hline Entreprises n'ayant pas adopté de mesures (n = 189239 ; n' = 1206) & $3,6 \%$ & $80,7 \%$ & $84,2 \%$ \\
\hline Entreprises ayant adopté au moins une mesure $\left(n=53805 ; n^{\prime}=257\right)$ & $9,3 \%$ & $75,2 \%$ & $84,5 \%$ \\
\hline Ensemble des entreprises $(n=243044 ; n '=1463)$ & $4,8 \%$ & $79,5 \%$ & $84,3 \%$ \\
\hline p-value & 0,0001 & 0,5371 & 0,1465 \\
\hline
\end{tabular}

établissements qui comptent plus de 500 salariés et ces établissements sont proportionnellement plus nombreux à avoir pris des mesures), l'effet mesuré est significativement moindre, avec une progression de $4,1 \%$ : de $3,6 \%$ pour les entreprises n'ayant pas adopté de mesures à $7,7 \%$ pour les entreprises en ayant adopté au moins une. En tenant compte des taux d'occupation des véhicules, nous aboutissons donc à une fourchette comprise entre 2,1 et 3,3\% quant à la progression du covoiturage en tant que passager. Vu le nombre limité d'entreprises ayant adopté des mesures en faveur du covoiturage $(18 \%)$, sa part modale ne progresse toutefois que de $1,2 \%$. Le nombre limité d'entreprises ayant adopté des mesures doit également nous pousser à la prudence concernant la robustesse de nos résultats. En effet, vu les faibles effectifs concernés par les mesures, il est possible que certains effets de composition n'aient pu être détectés (par exemple quant à la localisation ou quant au secteur d'activité), ce qui conduirait alors à une surestimation de l'impact réel.

Pour terminer avec le sujet du covoiturage, signalons encore que la progression de cette pratique semble bien se réaliser au détriment de la conduite individuelle et non au détriment d'autres modes que la voiture.

\section{Conclusion}

Le premier objectif de la recherche présentée dans cet article était d'évaluer l'impact de la gestion de la mobilité par les entreprises actives en Wallonie. Afin d'aboutir à cet objectif, nous avons comparé les pratiques de mobilité entre les établissements qui n'ont pas pris de mesures en vue de réduire la conduite automobile et les établissements qui ont développé une politique active. Sur le plan méthodologique, la difficulté de cette approche est de maîtriser l'influence des effets de composition. En effet, les entreprises ayant choisi d'adopter des mesures se situent généralement dans un contexte favorable pour le faire. Il est donc délicat d'établir des comparaisons «toutes choses égales par ailleurs ». Afin de maîtriser les effets de composition, nous avons établi divers scénarios en ayant préalablement segmenté l'échantillon disponible. Il ressort de ces simulations que considérer simultanément la localisation macro et la localisation micro des établissements est le mode de segmentation le plus efficace. Ce résultat tient vraisemblablement au fait qu'en considérant la localisation des établissements, nous utilisons indirectement une information très riche sur de nombreuses caractéristiques qui déterminent les comportements de mobilité.

$\mathrm{Au}$ final, des résultats mitigés découlent de nos analyses. D'un côté, les mesures en faveur des transports en commun et du covoiturage se montrent relativement bénéfiques sur la limitation de l'autosolisme. De l'autre, les mesures visant à favoriser la pratique cycliste ne semblent pas porter leurs fruits. Pour les transports en commun et le covoiturage, cette conclusion peut sans doute être généralisée à l'ensemble de la Belgique. Pour le vélo, la situation est par contre différente en Flandre. En effet, des travaux antérieurs ont montré que la pratique cycliste y sort renforcée lorsque certaines mesures sont adoptées, en particulier lorsque les employeurs acceptent de financer des indemnités qui couvrent les frais de déplacement de leurs salariés [12, 50]. En Wallonie, par contre, nos analyses indiquent que l'adoption de mesures en faveur du vélo entraîne un report depuis la marche plutôt que depuis la voiture.

Selon nos simulations, les mesures en faveur des transports en commun auraient conduit à une réduction de la part de l'automobile de $2,3 \%$. Les mêmes simulations montrent que l'impact pourrait atteindre $5,1 \%$ si l'ensemble des entreprises activait ce levier des mesures en faveur des transports collectifs. Rappelons que cet impact théorique maximal peut être appréhendé comme l'impact moyen accompagnant la prise de mesures à l'intérieur d'une unité d'établissement. Concernant les mesures en faveur du covoiturage, nos calculs concluent à une progression de la part de ce mode de $1,2 \%$, avec un potentiel maximal de $4,1 \%$ si l'ensemble des établissements menait une politique active. En tenant compte de la différenciation entre les rôles de conducteur et de passager, cette estimation conduit à une 
fourchette comprise entre 2,1 et 3,3\% quant à la progression potentielle du covoiturage en tant que passager.

En sommant l'impact des mesures en faveur du covoiturage et des TC, la diminution moyenne de l'autosolisme engendrée par la prise de mesures au sein d'une unité d'établissement est estimée entre 7,2\% et $8,4 \%$. Cette estimation est assez proche de l'ordre de grandeur des $6 \%$ mis en avant par Rye [7]. La convergence semble d'autant plus forte que nos estimations se basent sur l'hypothèse d'une maitrise parfaite des effets de composition. Cela n'étant vraisemblablement pas le cas, il est probable qu'elles surestiment les impacts et qu'elles soient donc optimistes par rapport à la réalité des évolutions comportementales. Du point de vue des entreprises qui activent des politiques visant à limiter l'autosolisme, il ressort de nos chiffres que viser une réduction de cette pratique comprise entre $6 \%$ et $8 \%$ s'apparente à un objectif à la fois réaliste et moyennement ambitieux.

En plus de chercher à quantifier l'impact des politiques de mobilité menées par les entreprises sur les parts modales des politiques de mobilité menées par les entreprises, l'objectif de cet article était également de préciser comment ces politiques interagissent avec l'organisation spatiale des territoires. Une de nos hypothèses était ici que le contexte spatial des centres-villes et des lieux qui bénéficient d'une bonne accessibilité par les alternatives à l'automobile représente un terreau favorable à la prise de mesures en faveur du report modal. Pourtant, nos calculs ne vérifient pas cette hypothèse. En effet, ils montrent que les mesures actuelles sont peu opérantes pour les entreprises situées dans le centre des agglomérations wallonnes. Notre interprétation de ces comportements « inflexibles » est que les incitations - en particulier financières - visant à favoriser le report modal ne sont pas assez puissantes pour modifier les habitudes des travailleurs qui, malgré l'excellente desserte des centres-villes par les TC, décident malgré tout d'utiliser la voiture afin d'y accéder. Dans le même ordre d'idée, nos développements indiquent que les mesures visant à favoriser le report modal depuis la voiture vers le train sont peu efficaces lorsque les travailleurs parcourent des distances importantes. En effet, à partir d'un certain seuil kilométrique, la voiture est délaissée par les navetteurs même si l'abonnement de train n'est pas remboursé.

Sans conteste, les bénéfices des politiques de gestion de la mobilité par les entreprises sur l'usage de l'automobile pourraient être plus importants. Nous pouvons ici nous appuyer sur les conclusions de Witlox et ses collègues lorsqu'ils soulignent que les politiques actuellement menées en Belgique sont insuffisamment intégrées [12]. Bien que les incitations financières, l'amélioration des infrastructures et la diffusion d'informations soient des leviers relativement efficaces, les entreprises hésitent souvent à prendre des mesures dissuasives telles que la limitation ou la tarification du stationnement. De plus, les mesures adoptées sont souvent considérées isolément. Leur influence sur les changements de comportement pourrait donc être plus importante si elles étaient envisagées comme des composantes d'une politique de mobilité intégrée [12]. Les mesures adoptées par les entreprises devraient également être mieux intégrées aux politiques publiques, tant pour les politiques cyclables que pour les choix opérés en matière de stationnement. Pour le vélo, rappelons que l'efficacité des mesures adoptées en Flandre tient à un contexte général bien plus favorable aux deux-roues. Concernant le report modal vers les transports collectifs, notre travail montre que l'effet dissuasif de la limitation du stationnement existe peu dans les villes wallonnes. Pour ces villes, des marges de manœuvre existent probablement en la matière. Les valoriser, en concertation avec les entreprises, devrait donc permettre d'y limiter la pression automobile.

En conclusion de cet article, nous voudrions aussi souligner l'importance d'une amélioration des dispositifs statistiques et de collecte de l'information. Recommandons d'abord la réactualisation de l'outil des cartes d'accessibilité, dont notre travail a clairement démontré l'utilité. De même, recommandons l'amélioration des prochains diagnostics relatifs aux déplacements domiciletravail qu'organise l'administration fédérale belge. En effet, il serait sans doute utile que les prochains questionnaires permettent de mieux distinguer l'utilisation de la voiture en tant que conducteur ou en tant que passager. Disposer de ce type d'informations permettrait, par exemple, de mieux préciser l'impact des mesures en faveur du covoiturage. En outre, les questionnaires devraient mieux prendre en compte tant les déplacements intermodaux que les pratiques multimodales.

Comme point final, nous voudrions souligner l'intérêt de poursuivre la recherche présentée dans cet article. Nous pensons tout d'abord à l'opportunité de la compléter par des méthodes plus qualitatives. Des enquêtes préparées spécifiquement pour investiguer le sujet de la gestion de la mobilité par les entreprises permettraient ainsi d'approfondir les résultats que nous venons de présenter. En la matière, nous pensons particulièrement à des enquêtes visant à mieux comprendre les comportements « inflexibles » observés sur les sites qui bénéficient de la meilleure accessibilité par les alternatives à l'automobile. En parallèle, nous pensons également à l'opportunité de transposer la méthode développée dans cet article à d'autres territoires. Comme nous l'avons vu, appliquer à la Wallonie une approche établissant des scénarios en fonction des contextes micro et macro-spatiaux a donné des résultats robustes. Établir le même type de simulations dans d'autres territoires devrait donc aussi être très riche d'enseignements. 


\section{Références}

1. Cornelis E (2012) Transport et mobilité. In Germain M, Robate R (eds.) L'état de la Wallonie. Portrait d'un pays et de ses habitants. Les éditions namuroises, Namur, pp 159-179.

2. Halleux J-M, Lambotte J-M (2006) Comment maitriser la demande en mobilité et limiter la dépendance automobile par l'aménagement du territoire ? Les Cahiers du Cepess 1: 23-43.

3. Patrice B (2000) La Gestion de la Mobilité et les Entreprises. Les enseignements de l'étranger : Exemples américains et européens. Transport, Environnement, Circulation 158:30-39.

4. Giuliano G, Wachs M (1992) Managing Transportation Demand: Markets versus Mandates. Reason Foundation 148:1-23.

5. European Commission (2009) Action Plan on Urban Mobility. Bruxelles, $12 \mathrm{p}$.

6. Posch K-H (2009) Management de la mobilité : une dynamique qui s'amplifie en Europe. Les Cahiers de l'IAU Ile-de-France 150: 96-99.

7. Rye T (2002) Travel plans: do they work? Transport Policy 9: 287298.

8. Bradfer F (2010) Mobilité et déplacements domicile-travail. CeMathèque 29.

9. De Witte A, Hollevoet J, Dobruszkes F et al. (2013) Linking modal choice to mobility: a comprehensive review. Transportation Research Part A: Policy and Practice 49: 329-341.

10. Vanoutrive T, Van Malderen L, Jourquin B et al. (2010) Mobility Management Measures by Employers: Overview and Exploratory Analysis for Belgium. European Journal of Transport and Infrastructure Research 10: 121-141.

11. Rye T (1999) Employer Transport Plans - A case for Regulation. Transport Reviews 19: 13-31.

12. Witlox F, Jourquin B, Thomas I et al. (2011) Assessing and Developing Initiatives of Companies to control and reduce Commuter Traffic “ADICCT”. Science for Sustainable Development, 103 p.

13. Van Malderen L, Jourquin B, Thomas I et al. (2012) On The Mobility Policies of Companies: What are the Good Practices? The Belgian Case. Transport Policy 21: 10-19.

14. Rye T, Ison S (2005) Overcoming barriers to the implementation of car parking charges at UK workplaces. Transport Policy 12(1): 57-64.

15. Ewing R, Cervero R (2010) Travel and the built environment. Journal of the American Planning Association 76(3): 265-294.

16. Dujardin S, Pirart F, Brévers F et al. (2012) Home-to-work commuting, urban form and potential energy savings: A local scale approach to regional statistics. Transportation Research Part A: Policy and Practice 46/7:1054-1065.

17. Le Néchet F, Aguiléra A (2012) Forme urbaine et mobilité domicile-travail dans 13 aires urbaines françaises : une analyse multiéchelle. Recherche Transports Sécurité 28:259-270.

18. Dickinson J-E, Kingham S, Copsey S, Hougie D-J-P (2003) Employer travel plans, cycling and gender: will travel plan measures improve the outlook for cycling to work in the UK? Transportation Research Part D: Transport and Environment 8/1:53-67.

19. Pucher J, Dill J, Handy S (2010) Infrastructure, programs, and policies to increase bicycling: an international review. Preventive Medicine 50:S106-S125.

20. De Witte A, Macharis C, Mairesse O (2008) How persuasive is 'free' public transport? A survey among commuters in the Brussels Capital Region. Transport Policy 15/4:216-224.

21. Kingham S, Dickinson J, Copsey S (2001) Travelling to work: will people move out of their cars. Transport Policy 8/2:151-160.

22. Hagman O (2003) Mobilizing meanings of mobility: car users' constructions of the goods and bads of car use. Transportation Research Part D: Transport and Environment 8:1-9.
23. Transit Co-operative Research Program (1994) Cost effectiveness of TDM programs: Working Paper 2. COMSIS Corporation, Maryland, USA.

24. Cairns S, Newson C, Davis A (2010) Understanding successful workplace travel initiatives in the UK. Transportation Research Part A: Policy and Practice 44/7:473-494.

25. SPF Mobilité \& Transports (2010) Diagnostic des déplacements domicile-travail 2008 - Rapport final. Bruxelles, 92 p.

26. Castets B (2000) Les plans de mobilité ou comment inciter aux changements de comportement pour les déplacements liés au travail. Transport, Environnement, Circulation 158:17-21.

27. Ligtermoet D (1998) Zeven jaar vervoermanagement: synthese van ervaringen Report to Adviesdienst Verkeer en Vervoer. Netherlands Ministry of Transport, The Hague.

28. Touwen M (1999) Travel Planning in the Randstad: An Evaluation Based on ReMOVE. Report to Netherlands Ministry of Transport, The Hague.

29. Nozick L-K, Borderas H, Meyburg A-H (1998) Evaluation of travel demand measures and programs: a data envelopment analysis approach. Transportation Research Part A: Policy and Practice 32/5:331-343.

30. Marsden G (2006) The evidence base for parking policies: a review. Transport Policy 13/6:447-457.

31. Limtanakool N, Dijst M, Schwanen T (2006) The influence of socioeconomic characteristics, land use and travel time considerations on mode choice for medium-and longer-distance trips. Journal of Transport Geography 14/5:327-341.

32. Vanoutrive T, Van De Vijver E, Van Malderen L et al. (2012) What determines carpooling to workplaces in Belgium: location, organisation, or promotion? Journal of Transport Geography 22:77-86.

33. Kaufmann V (2000) Mobilité quotidienne et dynamiques urbaines. La question du report modal. Presses Polytechniques et Universitaires Romandes, Lausanne, $251 \mathrm{p}$.

34. O'Fallon C, Sullivan C, Hensher D-A (2004) Constraints affecting mode choices by morning car commuters. Transport Policy 11/1:17-29.

35. Van Damme O (2010) Les plans de déplacements d'entreprise. Le CRR, votre conseiller pour l'élaboration des plans de déplacements d'entreprise. Centre de recherches routières 8 : $1-16$.

36. Hubert J-P (2004) Mobilité urbaine, périurbaine, rurale en Belgique: où sont les différences ? les Cahiers Scientifiques du Transport 45:83-100.

37. Van Hecke E (1998) Actualisation de la hiérarchie urbaine en Belgique. Bulletin du Crédit Communal 205/3:45-76.

38. Van Hecke E, Halleux J-M, Decroly J et al. (2007) Noyaux d'habitat et régions urbaines dans une Belgique urbanisée. Enquête socio-économique 2001. Monographie $\mathrm{n}^{\circ} 9$.

39. Daxhelet S, Lambotte J-M (2005) Mesurer l'accessibilité des lieux. In De Keersmaecker M-L (ed.) Protocole de Kyoto : aménagement du territoire, mobilité et urbanisme. Edition Etudes et Documents CPDT, Namur, pp 81-90.

40. Daxhelet D, Kessler L, Lambotte J-M et al. (2002) Etude complémentaire relative aux profils d'accessibilité - profils de mobilité, CPDT, Université de Liège, $76 \mathrm{p}$.

41. Cornet Y, Daxhelet D, Halleux J-M et al. (2005) Cartographie de l'accessibilité par les alternatives à la voiture. Les journées géographiques belges.

42. van Wee B, van der Hoorn T (1996) Employment location as an insturment of transport policy in the Netherlands. Fundamentals, instruments and effectiveness. Transport Policy 3/3:81-89.

43. Verhetsel A, Van Hecke E, Thomas I et al. (2009) Le mouvement pendulaire en Belgique. Les déplacements domicile-lieu de travail. Enquête socio-économique 2001. Monographie ${ }^{\circ} 10$. 
44. Vandermeer M-C (2010) Les déplacements domicile-travail en Wallonie. Le rôle de l'accessibilité et du profil des entreprises sur les répartitions modales. Mémoire de master en sciences géographiques. Université de Liège, inédit.

45. Bianchet B, Wertz I, Halleux J-M, Wayens B (2011) Les secteurs Les activités économiques et industrielles. In: Conférence Permanente du Développement Territorial (ed.) Diagnostic territorial de la Wallonie. Service Public de Wallonie, Namur, Belgique, pp 77-90.

46. De Schutter T (2002) Quels outils pour la gestion communale du stationnement ? In: Union des Villes et des Communes de Wallonue (ed.) La problématique du stationnement. Actes $d u$ Colloque du 12 décembre 2002, pp 48-52.

47. Brisbois X, Kouabenan D-R, Rubens L (2012) Mixité sociale et choix modal : importance des dimensions symboliques dans l'attrait des transports collectives. Recherche, Transports, Sécurité 28:181-189.

48. Vande Walle S, Steenberghen T (2006) Space and time related determinants of public transport use in trip chains. Transportation Research Part A: Policy and Practice 40/2:151-162.

49. Ye X, Pendyala R-M, Gottardi G (2007) An exploration of the relationship between mode choice and complexity of trip chaining patterns. Transportation Research Part B: Methodological 41/1:96-113.

50. Vanoutrive T, Van Malderen L, Jourquin B et al. (2009) Let the business cycle! Investigating the role of employers in the bicycle use of employees using a multilevel model. Belgeo 2:217-231.

51. Vandenbulcke G, Thomas I, de Geus B et al. (2009) Mapping bicycle use and the risk of accidents for commuters who cycle to work in Belgium. Transport Policy 16/2:77-87. 